\title{
Interactions, Goals and Rules in a Collaborative Modelling Session
}

\author{
Denis Ssebuggwawo ${ }^{1}$, Stijn Hoppenbrouwers ${ }^{1}$, \\ and Erik Proper ${ }^{1,2}$ \\ 1 Institute of Computing and Information Sciences, Radboud University Nijmegen \\ Heyendaalseweg 135, 6525 AJ Nijmegen, The Netherlands, EU \\ D.Ssebuggwawo@science.ru.nl, stijnh@cs.ru.nl \\ 2 Capgemini Nederland B.V., \\ Papendorpseweg 100, 3528 BJ Utrecht, The Netherlands, EU \\ e.proper@acm.org
}

\begin{abstract}
Collaborative modeling can enhance productivity and quality of modeling in system development and enterprise engineering projects by helping to construct agreement and a sense of model ownership among stakeholders/modelers. Most of these stakeholders have relatively low expertise in formal modeling; advanced modeler-oriented support for collaborative modeling is a possible remedy. As a basis for further development of such support (methods, tools), we have carried out a detailed exploratory study of the interaction between modelers, involving diverse aspects of modeling: goal setting, modeling language concepts, planning, etc. Central in our approach is the study of how collaborative modelers negotiate, set, use, and deal with the various rules/goals governing interactive modeling sessions. We describe the conceptual framework and approach used for our analysis, and present findings from a case study which focused on the first phases of a session concerning basic Business Process Modeling. We also compare our findings to some existing work, to demonstrate the relevance of our approach.
\end{abstract}

Keywords: Collaborative Modeling, Business Process Modeling, Quality of Modeling, Modeling as a Game.

\section{Introduction}

System development, and conceptual modeling in particular (including, under our broad definition, information modeling, process/workflow modeling, and even business rule modeling), is a process in which communication plays a vital role [1. In system development (including enterprise engineering) a number of stakeholders are usually brought aboard the system development ship with varying skills, expertise, and knowledge. This results in a heterogeneous group of stakeholders including, for example, project managers, (prospective) users who may act as domain experts, system architects, analysts, programmers, etc. In such environments, participants engage in various types of conversation during 
the creation of a agreed models. Such conversations involve negotiation, which results in accepts, rejects, modifications, etc., (see, for example, 23]).

Much has been written about (conceptual) modeling, mainly in the area of information systems. Some have developed frameworks to attain the desired qualities of the developed models [4.5]; others developed guidelines for modeling, see for example, 6]. Alternatively, we have worked toward understanding of the detailed process (act) of modeling; see for example [7. During the collaborative process of system development, stakeholders "move through a process in which they combine their expertise, insights and their resources to bring them to bear for the task at hand" 8]. The importance of involving different hierarchical level representatives in a (re-)engineering process is recognized in 9. However, the emphasis in the bulk of the literature is on tools and techniques used by the stakeholders in order to achieve the desired model quality (completeness and correctness). It is our contention, however, that when we are concerned with the quality of the final model, we also need to analyze the process that generates it. How a process is executed is a major influence (negatively or positively) on the quality of the model.

If the complex and dynamic collaborative interactions involved are not properly organized and supported, the benefits that potentially accrue from them may not be realized. This could, for example, be due to the limitations of the human mind, collaborative capacity, or of resources needed. To overcome some of these limitations, a number of approaches have been suggested, e.g. using professional facilitators in Group Support Systems $(G S S)$ and Group Decision Support Systems $(G D S S)$ [10], group model building in Systems Dynamics $(S D)$ [1], etc.

The work of Peter Rittgen [3] is closely related to our own, based on similar principles, and therefore particularly relevant to this paper. His Collaborative Modeling Architecture(COMA) tool [12 reflects a similar approach to collaborative system analysis and design. However, while he focuses on negotiation of models as such (which is indeed the core activity), he largely ignores other aspects (like language setting, planning, sub-model definition, etc.), or sets default choices for them. While we consider his approach a good start, we believe more differentiated and in-depth analysis of real modeling processes will contribute to a broader and deeper understanding of what concerns modelers when they do their thing, leading to refinement and improvement of the collaborative modeling methods and tools.

The purpose of this paper is to propose, and report on results of, an approach for analyzing the process (act) of modeling by analyzing the communication between the modelers in a facilitator-free environment. Our in-depth analysis aims to reveal conventions/rules for interaction and collaboration in modeling. Knowledge of such patterns contributes to our understanding of the process of modeling as it really occurs in operational modeling. Consequently, we hope to advance our capability to design effective, more "modeler-oriented" support tools for collaborative modeling processes. Such tools should actively support the ways of thinking and interacting that lead to fulfillment of clear modeling 
goals [13. This goes beyond the capabilities of the highly technical "modeloriented" editors that are currently the best we can offer modelers. In order to operationalize our approach, we hypothesize that the interactions that take place in collaborative modeling sessions can be looked at as games with players who may either explicitly or implicitly determine and play by rules of a modeling game. Taking this metaphorical gaming perspective helps us to home in on the rules and goals driving the modeling process, some of which may later feed requirements for support tools.

In the remainder of this paper, we explain our operational conceptual framework and methodological approach concerning the approach sketched above. We illustrate the framework and approach at the hand of data and results from a case collaborative modeling session. The main results are interaction and rule categories found (reflecting various concerns of modelers), and various relevant observations. We will also compare our findings with some existing work, mostly to demonstrate the relevance of our approach.

\section{Analytical Setup}

In this section we give a brief overview of the questions we try to answer (in view of our case study) in this paper. We then present an operational methodology designed to help us find answers to the questions raised. As discussed, we take the view that (conceptual) modeling in system and enterprise engineering can be regarded as a communicative process in which the modelers participate. We therefore mainly look at (categories in) interactions between modelers. This in turn helps us examine the rules and goals under which the development takes place. Crucially, the setting of rules and goals may itself be subject to interaction. Consequently, the rules can be changed and extended during the game, as part of the game.

\subsection{Research Questions}

We raise the following questions which motivate our way of thinking in this paper:

- What are modelers concerned with during the case modeling session?

- What are the main categories of the rules governing a (process) modeling session?

- How do rule categories found in the case study relate to the categories as proposed and used in previous relevant frameworks for analysis? (see Sect. 3 and Sect. 4.5 for details)

- What further observations can we make concerning rules, rule setting, and interaction?

\subsection{General Set-Up of the Study}

We created a fairly elaborate context and asked three subjects to create a process model together. 
Assignment. Prior to the session, we provided the modelers with an elaborate written domain context (and enough time to read and digest it), even though we expected them not to get very far in producing an actual model (given the limited time available). The assignment we gave them was to start process modeling and see how far they could get, with the added requirement that they should agree on the outcome. We very deliberately did not dictate the use of a modeling language, but since the modelers had some experience in the use of UML Activity diagrams, we expected they would use this language (which indeed they did). Part of our interest is in how modelers deal with (agreeing about) modeling concepts to use. The business process scenario given to the modelers was based on a real life case, and concerned the development of a Hazardous Material Management System by the Materials Management Department of a city council through its Management Information Systems Department.

Researchers. The modeling session was organized and passively attended by two researchers. They took care of briefing and debriefing, observing and notetaking. One helped clarify a few concepts in the scenario description when this became necessary (i.e. he briefly acted as an informant, supplementing the written scenario provided before the session). Importantly, the researchers did not act as facilitators: the entire modeling session as such was intentionally left in the hands of the modelers.

Modelers. Three modelers participated in the actual session. They all had some experience in process modeling in a system development context, but were not expert modelers.

Recording the basic data. The session (which took 18 minutes) was video recorded with good sound quality. The modelers were also given a digital writing pad, which was recorded alongside the video. This provided us with a full, synchronized recording of all raw data we could wish for. See Fig. 1 for a snapshot of the recording.

Transcription. A complete transcription was made of the recording, including a detailed description of the drawings on the pad (which we consider a form of utterance, on par with verbal utterances). Table 1 shows a sample of the transcript. The three modelers are called M, D and R.

Data structuring and coding. To effectively study the conversation patterns in the modeling session, we identified atomic interactions (i.e., disentangled them if they were wrapped up in complex sentences) and annotated and categorized them. Table 2 shows an example of annotated and categorized data for the raw conversational data from Table 1. As usual in qualitative research with an explorative flavor, finding an optimal coding system is part of the effort. Hence, the coding system presented was in fact refined in the course of the analysis.

For the basics of our approach, we drew mainly on Language-Action Perspective (LAP) theory and Discourse Analysis, see for example [14. The basic annotation structure will be discussed in the next section. 
Table 1. Sample transcript of conversations from the video recording

\begin{tabular}{|c|c|c|}
\hline Time & Actor & Speech Act \\
\hline $00: 34$ & $\mathrm{M}$ & OK, we have to model...Process...Where shall we start? \\
\hline 00:41 & $\mathrm{D}$ & Which language? \\
\hline 00:44 & $\mathrm{M}$ & $\begin{array}{l}\text { Hah. May be we just first...something on the fly, some arrows and } \\
\text { some blocks, and then work it out later? }\end{array}$ \\
\hline 00:52 & $\mathrm{D}$ & Then we have to draw it twice, but...(laughs) \\
\hline 00:55 & M & So, What is the main process? \\
\hline 00:59 & $\mathrm{D}$ & $\begin{array}{l}\text { Those are here (laughs, points to the document), so may be swim } \\
\text { lane diagrams }\end{array}$ \\
\hline 01:08 & $\mathrm{R}$ & Yes, could be. \\
\hline 01:10 & $\mathrm{M}$ & So as first ordering we see things ordering, dispensing and disposing \\
\hline 01:15 & $\mathrm{D}$ & Yes, so 5 swim lane diagrams \\
\hline 01:17 & M & So is it necessary for each process? Draw all five? \\
\hline $01: 21$ & $\mathrm{D}$ & I guess so. Maybe you get short diagrams, but..... \\
\hline $01: 25$ & $\mathrm{M}$ & $\begin{array}{l}\text { Or we can make five first, so one for ordering, one for receiving, one } \\
\text { for storing.... }\end{array}$ \\
\hline 01:30 & $\mathrm{D}$ & Yes? \\
\hline $01: 32$ & $\mathrm{R}$ & That's what he meant I think. \\
\hline
\end{tabular}

Table 2. Sample coding and categorization of transcribed data

\begin{tabular}{|c|c|c|c|c|}
\hline Time & Topic & Topic\# & Categorization & Response to \\
\hline $0: 34$ & Set Creation Goal & 1 & Proposition-[We must make a process model] & \\
\hline & Set Planning & 2 & Question-[Where shall we start?] & \\
\hline $0: 41$ & Set Grammar Goal & 3 & Question-[Which language?] & \\
\hline $0: 44$ & Set Grammar Goal & 4 & Proposition/Answer-[Blocks and arrows] & 3 \\
\hline & Set Planning & 5 & $\begin{array}{l}\text { Proposition/Answer } \\
\text { [First do something on the fly, then work it out later] }\end{array}$ & 2 \\
\hline $0: 52$ & Set Planning & 6 & $\begin{array}{l}\text { Argument-against } \\
\text { [But then we have to draw it twice] }\end{array}$ & 5 \\
\hline $0: 55$ & Set Content & 7 & Question -[What is the process?] & \\
\hline 0:59 & Set Grammar Goal & 8 & $\begin{array}{l}\text { Proposition-Counter } \\
\text { [Let's use swim lanes] }\end{array}$ & 5 \\
\hline 1:08 & Set Grammar Goals & 9 & Agreement-[Yes] & 8 \\
\hline $1: 10$ & Set Content & 10a & $\begin{array}{l}\text { Proposition } \\
\text { [We have Processes, Ordering, Dispensing and Disposing] }\end{array}$ & \\
\hline 1:15 & $\begin{array}{l}\text { Set Content } \\
\text { Set Creation Goal }\end{array}$ & $\begin{array}{l}10 b \\
10 c\end{array}$ & $\begin{array}{l}\text { Agreement-[Yes] } \\
\text { Proposition-[Let's create } 5 \text { swim lane diagrams] }\end{array}$ & $10 a$ \\
\hline 1:17 & Set Creation Goal & 11 & $\begin{array}{l}\text { Question-Doubt } \\
\text { [Draw } 5 \text { swim lane diagrams for each of the } 5 \text { processes?] }\end{array}$ & $10 a$ \\
\hline $1: 21$ & Set Creation Goal & 12 & Answer-[Yes] & 11 \\
\hline & Set Creation Goal & 13 & $\begin{array}{l}\text { Argument for } \\
\text { [May be you get short diagrams but still this is the way to go] }\end{array}$ & $10 \mathrm{c}$ \\
\hline 1:25 & Set Creation Goal & 14 & $\begin{array}{l}\text { Proposition, Agreement } \\
\text { [Let's create } 5 \text { swim lane diagrams] }\end{array}$ & $10 a$ \\
\hline 1:30 & Set Creation Goal & 15 & $\begin{array}{l}\text { Argument-Proposition } \\
\text { [Yes, Isn't that what I just proposed?] }\end{array}$ & 14 \\
\hline $1: 32$ & Set creation Goal & 16 & Agreement, Question [Would 14 be OK?] & 14 \\
\hline
\end{tabular}




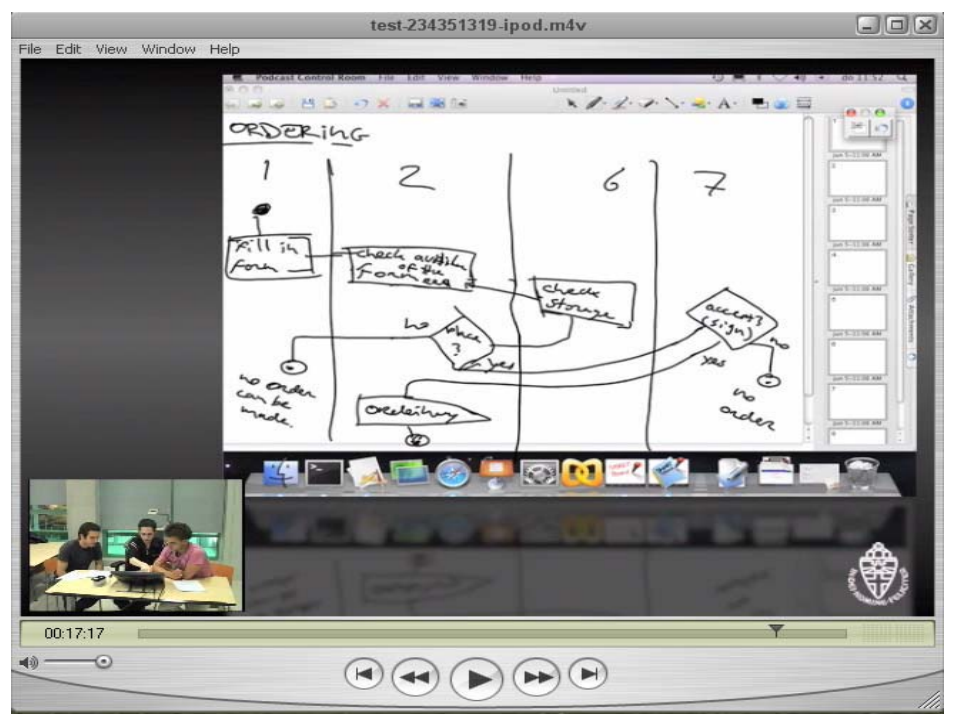

Fig. 1. Snap-shot of the recording

\section{$3 \quad$ Framework and Concepts}

In this section, we explain and demonstrate our analytical framework and approach at the hand of some selected fragments of the case; presenting the complete case analysis would take too much space. Our conceptual framework for analysis is based on previous theoretical work on the act of modeling, but pushes for operationalization of the theory in the form of qualitative analysis of (transcripts of) actual modeling sessions. It is rooted in discourse analysis, but extends into the definition and deployment of rules representing conventions and agreements concerning models and modeling processes.

In a collaborative modeling session modelers come together to perform some modeling task (for example, the creation of a business process diagram in some domain, for some purpose). They interact and communicate their ideas and opinions to other members. For them to reach consensus and agreement, they need to commit themselves to work as a team and abide by their collective knowledge, conventions and decisions (rules of their game). Their communication strategy sets the goals and rules (explicitly or implicitly) for a conversational dialog in which the modelers propose and argue about (negotiate) the different positions raised. This communication may result in (dis)agreement with, and acceptance/rejection of, the ideas proposed. The interplay between interactions, rules, and models, as discussed in our framework, is depicted in Fig. 2

As mentioned, modelers are guided and restricted by modeling rules and goals. In fact, we view goals as a key type of rule ("goal rules"): from a gaming perspective, the goals are rules setting states to strive for. The rules should ideally 


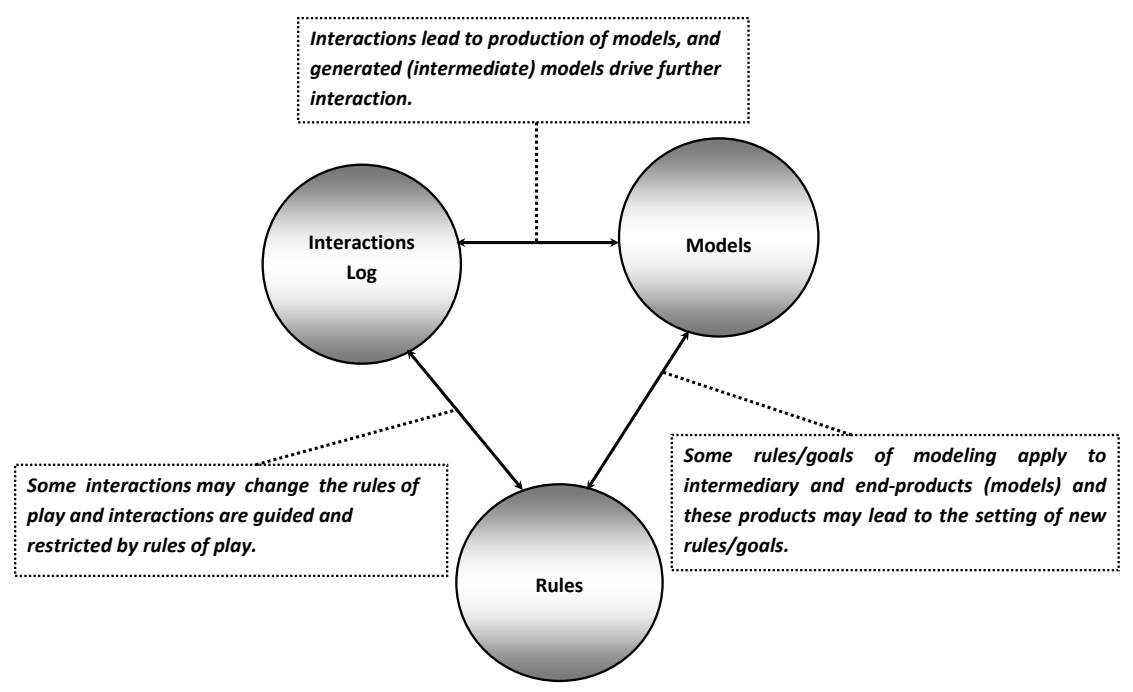

Fig. 2. A methodological approach for analyzing interactions, rules and models

guarantee process and model quality, but they also reflect existing conventions for (inter)action in modeling and conversation. We distinguish two basic types of rules in collaborative modeling: rules set for the game, i.e. setting the game as such, and rules set in the game. i.e. by the players. These rules can further be classified as either explicit or implicit rules. The combined distinctions form a simple $2 \times 2$ matrix. We consider all four resulting categories crucial in understanding "how people model" in terms of "the rules they play by", but focus on explicit rules. In our case study, the explicit rules set in the game outnumber the ones set for the game.

In order to be able to analyze a conversation in terms of specialized concepts (a form of qualitative text analysis), we need a coding system to be applied to the recorded interactions. In addition, we make explicit a set of rules governing the interactions and the products thereof. Finally, we can relate the product of the conversation, the model (and its consecutive versions), to the conversation, and to the rules where relevant; however, in the current paper we focus on rule setting as such.

- Interactions - with properties: time and interaction number, actor(player), topic/content, and speech act type. Table 3 gives an example of interaction coding, interpretation and the meta-data associated with its properties.

- Rules - with properties: time of activation, content and number of interaction it was activated in, time of deactivation, content and number of interaction it was deactivated in, type of rule. Interactions are identified by numbers. See Table 4 .

- Models - These are generated lists of propositions (statements) derived from the entire conversation up to some time $t$, and subject to selection criteria 
Table 3. Interaction coding, interpretation and meta-data

\begin{tabular}{|c|l|l|l|}
\hline Time/Int\# & Actor & Speech Act - [Type] & Topic \\
\hline \hline $6: 04105$ & $\mathrm{M}$ & $\begin{array}{l}\text { Should we introduce a vendor, actor 9? [Ques- } \\
\text { tion] }\end{array}$ & Set content \\
\hline \hline $6: 08106$ & $\mathrm{D}$ & $\begin{array}{l}\text { The material handler already functions as the } \\
\text { vendor. [Argue against 105] }\end{array}$ & Set content \\
\hline
\end{tabular}

Table 4. Rule coding, interpretation and meta-data

\begin{tabular}{|c|l|l|l|l|}
\hline Time $_{[A]}$ & Interaction $_{[A]}$ & Time $_{[D]}$ & Interaction $_{[D]}$ & RuleType \\
\hline \hline $14: 24$ & $\begin{array}{l}\text { Decisions are repre- } \\
\text { sented by means of a } \\
\text { diamond symbol. }[236]\end{array}$ & - & $\begin{array}{l}\text { Grammar } \\
\text { goal rule }\end{array}$ \\
\hline
\end{tabular}

Where Interaction ${ }_{[A / D]}$ is the interaction in which the rule is activated/de-activated. $\operatorname{Time}_{[A / D]}$ is the activation/de-activation time of the rule. However, there were no occurrences of rule de-activation in the case study.

Table 5. Model coding, interpretation and meta-data

\begin{tabular}{|l|l|l|}
\hline Time & Propositions & Selection Criteria \\
\hline \hline 12:23 & $\begin{array}{l}\text { Check Storage is the first activity in } \\
\text { swim lane 6. }\end{array}$ & $\begin{array}{l}\text { Proposed and drawn, not explicitly } \\
\text { disagreed with. }\end{array}$ \\
\hline 14:01 & And here the Item is finally ordered & Explicitly agreed with by all \\
\hline
\end{tabular}

determining which proposals make it to the common (shared) model. Table 5 shows two example propositions and the criteria that were used in selecting them for the model.

Viewing modeling as a game requires identification of the rules under which the modeling game is played, including goals driving and motivating the players (i.e., modelers). Previous work has dealt with identification of modeling goals based on known aspects of quality of modeling, concerning both the process of modeling and the models themselves. Our goal types were initially derived from the QoMo framework [5], in turn derived from the Semiotic Quality framework (SEQUAL) 4, but were now tested in practice, and thus a result of this paper is partial validation of the QoMo framework. Another link with an existing approach is with the COMA approach of Peter Rittgen [3] ; we will also briefly compare our findings with COMA. Such comparison is interesting as such but it also serves to demonstrate how our approach may contribute to the method and evaluation.

\section{Findings}

In this section we present our findings and observations; however, note that a different result is the analytical approach as such, as presented in this and the previous section. 
Table 6. Duration and number of interactions of the collaborative modeling phases

\begin{tabular}{|c|l|l|l|}
\hline Phase & Phase Activity & $\begin{array}{l}\text { Duration } \\
\text { (seconds) }\end{array}$ & $\begin{array}{l}\text { No. } \\
\text { Interactions }\end{array}$ \\
\hline I & $\begin{array}{l}\text { Setting of the main approach: choosing the lan- } \\
\text { guage and sub-division of work }\end{array}$ & 21 \\
\hline II & $\begin{array}{l}\text { Exploring and deciding which actors play a role } \\
\text { in the first partial process model. }\end{array}$ & 414 & 126 \\
\hline III & Modeling the sub-process & 527 & 144 \\
\hline \multicolumn{2}{|c|}{ Total } & $\mathbf{1 0 4 5}$ & $\mathbf{2 9 1}$ \\
\hline
\end{tabular}

We applied the basic framework and approach shown in Fig. 2 to the case study described in Sect. 2.2. Our central aim was to discover and make explicit the "rules of the modeling game", and the dynamics of them being set in view of particular goals and situations. The whole collaborative modeling session consisted of a total of 291 interactions and took 17.25 minutes or 1045 seconds. It showed three clearly distinguishable phases (see Sect. 4.4), each with its own typical proportion of interactions types. Table 6 summarized these phases.

\subsection{Categorization of the Speech-Acts}

In line with [3], it can be noted from the video and the transcription that the communication among the modelers can broadly be categorized as a negotiation. I consists of argumentations (argue for/against) by the modelers which results in either acceptance/agreement by all modelers, or rejection of the proposals. Explicit agreement only occurs at some points in the negotiations, whereas "silence means agreement" is the convention applied most in the case conversation. Rejection may come explicitly, as a result of a disagreement (objection) to a proposal or as a result of an agreement to drop a proposal. For our full set of speech act categories, see Table 7 which also gives the distribution of the interactional speech acts over the three phases.

Table 7. Number and type of speech acts within the phases

\begin{tabular}{|l|ll|lr|lr|lr|l|}
\hline Speech Act Type & \multicolumn{2}{|l|}{ Phase I } & \multicolumn{2}{|l|}{ Phase II } & \multicolumn{2}{|l|}{ Phase III } & \multicolumn{2}{|l|}{ Total } \\
\hline \hline & $\#$ & $\%$ & $\#$ & $\%$ & $\#$ & $\%$ & $\#$ & $\%$ \\
\hline Propose/Answer & 7 & 33 & 30 & 24 & 39 & 27 & 76 & 26 \\
\hline Counter_propose & 0 & 0 & 3 & 2 & 6 & 4 & 9 & 3 \\
\hline Question & 7 & 33 & 25 & 20 & 16 & 11 & 48 & 16 \\
\hline Argue_for & 2 & 10 & 3 & 2 & 7 & 5 & 12 & 4 \\
\hline Argue_against & 1 & 5 & 9 & 7 & 7 & 5 & 17 & 6 \\
\hline Agree_with/Accept & 4 & 19 & 17 & 13 & 23 & 16 & 44 & 15 \\
\hline Disagree_with/Reject & 0 & 0 & 16 & 13 & 7 & 5 & 23 & 8 \\
\hline $\begin{array}{l}\text { Non-verbal(graphical) } \\
\text { acts }\end{array}$ & 0 & 0 & 23 & 18 & 39 & 27 & 62 & 21 \\
\hline \multirow{2}{*}{ Total } & $\mathbf{2 1}$ & & & & & & & \\
\hline
\end{tabular}




\subsection{Categorization of Topics of Interactions}

All interactions either contribute toward the setting of a goal or toward goal fulfillment. Interactions of one type can fulfill several goals at the same time; for example, content setting should respect grammar rules and thus fulfills grammar goals, but content setting also, and primarily, fulfills creation goals. Interactions either set some proposition, or else concern one: they ask a question about one, argue for or against it, agree with it/accept it or disagree with/reject it. Also, they may draw one (on the digital pad). Accepted propositions set either rules or content. Accepted content becomes part of the model (see Table 5). In Table 8 we show the interaction topics as identified. The numbers and their corresponding percentages in the column total indicate the frequency use of the interaction topics.

Table 8. Number and type of interaction topics

\begin{tabular}{|c|c|c|c|c|c|c|c|c|c|c|c|c|}
\hline \multicolumn{13}{|c|}{ Interaction Topic } \\
\hline Phase & GRM & & & & $\mathrm{CON}$ & & $\mathrm{CR}$ & & $\mathrm{CC}$ & & Tot: & \\
\hline & \# & $\%$ & \# & $\%$ & \# & $\%$ & \# & $\%$ & \# & $\%$ & \# & $\%$ \\
\hline $\mathrm{I}$ & 4 & 25 & 3 & 43 & 2 & 1 & 12 & 100 & 0 & 0 & 21 & 7 \\
\hline II & 2 & 13 & 1 & 14 & 120 & 47 & 0 & 0 & 3 & 100 & 126 & 43 \\
\hline III & 10 & 63 & 3 & 43 & 131 & 52 & 0 & 0 & 0 & 0 & 144 & 49 \\
\hline Total & 16 & & 7 & & 253 & & 12 & & 3 & & 291 & \\
\hline
\end{tabular}

GRM = Grammar, $P L N=$ Planning, CON $=$ Content, $C R T=$ Creation, $C O L=$ Collaboration

The topics of the interactions mostly cover the categories proposed in [5], with some exceptions (see Sect.4.5). Grammar and creation goal setting were found as expected, as was content setting (which does not concern goal setting but fulfillment of goals). Collaboration Setting is an interaction category not previously proposed. It concerns how modelers are to collaborate with each other: what roles, hierarchy, responsibilities; how they organize themselves. Rules and goals may well be set for collaboration, but in the case this did not happen explicitly (though collaboration was topic of conversation). Another "new" topic was found: Planning Setting, concerning options for temporal scheduling and strategies concerning the fulfillment of creation goals. One other category was found but discarded in the tables because of its odd nature but may yet be interesting: Help Setting, concerning rules for asking "external help" (for example, asking for additional domain info). Categories like collaboration setting, planning, and even help setting are noteworthy and deserve some extra study; however, in the current case study they played very minor roles in explicit conversation.

\subsection{Rules and Goals}

As mentioned before, some rules were set for the game by the researchers, some set in the game, by players. All rules encountered were goal rules (e.g. creation 
goal rules or grammar goal rules). A special class of goal rule is a goal setting goal rule: it drives the modelers to set some explicit goal(s). Below we present the three goal rules that were explicitly set for the game (i.e in the assignment given): a creation goal, a grammar goal setting goal, and a validation goal. Next we present all rules set in the game. They mainly concern the modeling language (which concepts to use: grammar goals), and in one case how to divide the main task into sub-tasks and sub-models (an agreed refinement of the assigned creation goal).

These rules were set for the game:

1. Goal setting rule: creation goal. Content: "Create process model". Proposed and activated in the Assignment.

2. Goal setting goal rule: grammar goal. Content: "Set grammar goals". Proposed and activated in the Assignment

3. Goal setting rule: validation goal. Content: "All participants should agree on the model". Proposed and activated in the Assignment

These rules were set in the game:

1. Goal setting rule: grammar goal. Content: "Use blocks and arrows to represent activities and the relations between them". Proposed at $\mathrm{t}=0: 44$ and activated at $\mathrm{t}=8: 53$

2. Goal setting rule: grammar goal. Content: "Use Swim Lanes". Proposed at $\mathrm{t}=0: 59$ and activated at $\mathrm{t}=2: 21$

3. Goal setting rule: creation goal. Content: "There should be 5 swim lane diagrams composing the process model". Proposed at $\mathrm{t}=1: 15$ and activated at $\mathrm{t}=1: 42$ (by explicit agreement)

4. Goal setting rule: grammar goal. Content: "Use numbers above the swim lanes corresponding to the actors involved in the process". Proposed at $\mathrm{t}=2: 10$ and activated at $2: 21$

5. Goal setting rule: grammar goal. Content: "Sequences of activities are started with the START symbol". Proposed at $\mathrm{t}=8: 34$ and activated also at $\mathrm{t}=8: 34$ (used without discussion, but explicitly agreed on immediately).

6. Goal setting rule: grammar goal. Content: "Use end symbol to mark end of process flow". Proposed at $\mathrm{t}=14: 6$ and also activated at $\mathrm{t}=14: 06$

7. Goal setting rule: grammar goal. Content: "Decisions are represented by means of decision diamonds". Proposed at $\mathrm{t}=14: 18$ and activated at 15:19 (after considerable discussion, and against UML standard; participants are aware of this).

\subsection{Overall Findings and Observations}

In this section we focus on answering the questions raised in Sect. 2.1. Generally, we observe that in a group modeling session, modelers indeed go through a structured and highly interactive negotiation-like process guided by goals/rules that are either set for the game or set in the game. The modelers were mainly 
concerned with structuring the modeling process, modeling concept selection, and content setting, though other topics arose. In some cases, goals set early (for the game, in the assignment) were later refined, in particular the creation goal. Some effort also went into setting the grammar goals, as required by the initial "goal setting goal". A relatively low number of rules set for the game is followed by a larger number set in the game. It would of course be interesting to see what happens if more rules are set in the assignment, and indeed we intend to look at this in later experiments.

As shown in Table 6, at the hand of observed distribution of interaction topics we were able to distinguish three phases in the case session: 1) Setting the main approach -choosing the language and sub-division of work, 2) Exploring and deciding which actors play a role in the partial modeling process and 3) Modeling the subprocess. In view of our focus on "the rules of the game", the first phase can be seen as dedicated to such rule setting, whereas in the two other (main) phases, sporadic rule setting as required by the situation occurred. We conclude that two modes of rule setting seem to occur: planned, pro-active rule setting (phase I) and ad hoc, reactive rule setting (phases II and III).

In Table 7 and Table 8 we presented the categories and patterns of observed interactions (speech act types and interaction topics, respectively). The speech act types observed largely fit standard speech act categorization as common in the literature, as expected. We were able to finalize a small set of speech acts that enables us to elegantly code all interactions within scope of our analysis. As for interaction topics, these can broadly be put in the following categories: Planning, Creation, Grammar, Collaboration, and Content. Content concerns the model as such, the other topics concern matters of process and method. As shown in Table 8, content setting has by far the biggest share in the number of interactions (253), which was to be expected.

\subsection{Comparison with Existing Frameworks}

In this section we compare our framework and analysis to two relevant approaches from the literature: QoMo [5] and COMA [312].

Comparison with Quality of Modeling framework (QoMo). The QoMo framework involves an analysis of aspects for quality-of-modeling based on the product-oriented SEQUAL framework [4. Roughly speaking, QoMo rephrases the SEQUAL aspects (and some additional ones) as "goals for modeling". The QoMo Goals are theoretical in nature; our case study provides an opportunity for a reality check on QoMo. We will simply compare the QoMo goals-for-modeling from [5] (which is the most mature version) with the concerns-for-modeling that transpired from our close study of explicit interactions in an actual modeling session.

QoMo distinguishes Usage Goals, Creation Goals, Validation Goals, Argumentation Goals, Grammar Goals, Interpretation Goals, and Abstraction Goals. Usage goals are not explicitly encountered in our case study, as they are out of its scope, but implicitly they are part of the assigned domain description (Sect. 2.2) which provides a rough use context for which the process model is intended. 
Creation goals were clearly and explicitly encountered in the case study: a rough one was set for the game, and some refinement took place in the game (agreed setting of five sub-models). A validation goal was also explicitly set in the assignment, but no validation goals were discussed in the game, i.e. the plain initial goal "agree on the model" appears to have been workable for the modelers. Validation goals thus seem relevant enough, but in informal or preliminary settings their finer points remained implicit. Our assumption is that the modelers fall back on generic conventions for conversation and argumentation. However, we still expect validation goals to require refinement and specification in later stages of modeling (for example, when formal commitment of stakeholders comes in); further research will have to confirm this. Argumentation goals as well as interpretation goals are specializations of validation, and they too are not made explicit in the case study, yet again seem implicitly present as part of regular conventions for interaction. Interpretation, however, does seem to play some explicit role in content setting: phase II of the case mostly concerns attempts of the modelers to get a grip on poorly understood domain terminology; differences in meaning are discussed at length, and finally resolved - up to a point. This aspect also warrants further research. Grammar goals are clearly and explicitly set and used in the case study, which was in fact encouraged as the assignment included a "goal setting goal" that instructed the modelers to choose modeling concepts, hence to set grammar goals. Grammar goals setting thus proves a viable concept, and it is interesting to see how gradual, incremental introduction of concepts took place. Also, in more than one respect the modelers consciously diverted from standard UML (their initial choice). Abstraction goals, an obscure category as it is, were not encountered explicitly.

In addition (as discussed in Sect.4.2), several topics of interaction were identified that suggest extension of the theory-based QoMo goal set: Planning, Collaboration, and possibly also Help goals. However, they are arguably not directly quality-oriented, and hence this finding seems not so much to point out a gap in QoMo but rather the somewhat insufficient scope of a strict quality-oriented perspective on modeling goals.

\section{Comparison with the Collaborative Modeling Architecture (COMA).}

COMA is an interactive and collaborative modeling approach and tool which can be viewed as incorporating and thus setting various modeling goals/rules and interaction mechanisms, some of them as options, some of them "hard". Looking at the COMA tool [12] (its initial incarnation), the following rules are built into the system.

The tool is based on a standard UML editor for 5 types of diagram, including activity diagrams. This means that the Grammar Goals are hard-coded (though use of advanced concepts is often optional). In terms of the modeling language, therefore, our case study would probably have looked different if it had been conducted using the COMA tool: use of non-standard UML, like decision diamonds in an activity diagram, would have been impossible (which is not to say this would have presented the modelers with a problem). The other relevant goal category is that of Validation Goals. Rittgen built in support for validation 
in the form of an acceptation mechanism with decision parameters. This boils down to offering a choice out of various popular decision mechanisms observed to occur in collaborative modeling: a choice of detailed validation rules. In other words, COMA has a Goal Setting Goal underlying the validation parametrization (preceding a session). Finally, COMA is negotiation-oriented and supports argumentation for or against (partial) model diagrams. This is of course closely related to our speech act categories, and even amounts to the setting of an Argumentation Goal. All in all it seems that indeed, COMA comes close to embodying the main modeling goals as recognized in our approach and case. However, COMA is relatively restrictive in setting some main goals (so some refinement should be useful), and further ignores other aspects, like interpretation (negotiation about meaning), collaboration (team organization) and planning (delivery and task decomposition). Findings from this paper, but mostly from further research in a similar vein (Sect. 5), may well provide valuable input for evolutionary development of tools like COMA.

\section{Conclusions and Further Research}

We have presented and illustrated a research approach aimed at analyzing the detailed process (act) of modeling. We analyzed an actual collaborative modeling session. We presented a conceptual framework and a methodological approach. Findings were also presented, answering our research questions within scope of the case study. We also used our findings to perform a partial validation of the QoMo and COMA approaches, and thus demonstrated its applicability for evaluation purposes.

We do not claim that our approach is definitive and static. There clearly is ample room for elaboration and improvement. Similar analyzes for different (in particular, more restrictive) modeling contexts should be performed, which will no doubt require refinement of the method. Still, we hope to have shown that the sort of analysis presented can be fruitful, in particular in view of (empirical), HCI-style research into modeler-oriented, collaborative creation of models. In the near future, we plan to carry on in this line of work in a $\mathrm{PhD}$ project [15. that this paper is also a product of. Our main aim is to lay a foundation for the evaluation and design of advanced, modeler-oriented support tools for collaborative modeling.

\section{References}

1. Veldhuijzen van Zanten, G., Hoppenbrouwers, S.J.B.A., Proper, H.A.: System Development as a Rational Communicative Process. Journal of Systemics, Cybernetics and Informatics 2(4), 47-51 (2004)

2. Hoppenbrouwers, S.J.B.A., Proper, H.A., Weide, T.v.d.: Formal Modelling as a Grounded Conversation. In: Goldkuhl, M., Lind, G., Haraldson, S. (eds.) Proceedings of the 10th LAP Conference (LAP 2005), Kiruna, Sweden, pp. 139-155. Linkpings Universitet and Hogskolan I Boras, Linkping (2005) 
3. Rittgen, P.: Negotiating Models. In: Krogstie, J., Opdahl, A.L., Sindre, G. (eds.) CAiSE 2007. LNCS, vol. 4495, pp. 561-573. Springer, Heidelberg (2007)

4. Krogstie, J., Sindre, G., Jorgensen, H.: Process Models Representing Knowledge Action: A Revised Quality Framewok. EJIS 15, 91-102 (2006)

5. van Bommel, P., Hoppenbrouwers, S.J.B.A., Proper, H.A., Roelefs, J.: Concepts and Strategies for Quality of Modeling. In: Halpin, T.A., Krogstie, J., Proper, H.A. (eds.) Innovations in Information Systems Modeling, ch. 9. IGI Publishing, Hershey (2008)

6. Becker, J., Rosemann, M., von Uthmann, C.: Guidelines of the Business Process Modeling. In: van der Aalst, W.M.P., Desel, J., Oberweis, A. (eds.) Business Process Management: Models, Techniques, and Empirical Studies, pp. 30-49. Springer, Heidelberg (2000)

7. van Bommel, P., Hoppenbrouwers, S.J.B.A., Proper, H.A.: QoMo: A Modeling Process Quality Framework Based on Sequal. In: Proper, H.A., Halpin, T., Krogstie, J. (eds.) Proceedings of EMMSAD 2007, pp. 118-127. Tapir Academic Press, Norway (2007)

8. Vreede, G.J., de Briggs, R.O.: Collaboration Engineering: Designing Repeatable Processes for High-Value Collaborative Task. In: Proceedings of the 38th HICCS Conference, p. 17c. IEEE, Los Alamitos (2005)

9. Dean, D., Orwig, R., Lee, J., Vogel, D.: Modelling with a Group Modelling Tool: Group Support, Model Quality and Validation. In: System Sciences 1994: Collaboration Technology Organizational Systems and Technology: Proceedings of the 20th HICCS Conference, vol. 4, pp. 214-223. IEEE, Los Alamitos (1994)

10. Bostrom, R.P., Clawson, V.K., Anson, R.: Group Facilitation and Group Support Systems. In: Jessup, L.M., Valacinch, J.S.M. (eds.) Group Support Systems: New Perspectives, pp. 146-168. Macmillan, New York (1993)

11. Rouwette, E., Vennix, J., Van Mullekom, T.: Group Model Building Effectiveness. A Review of Assessment Studies. Sys. Dynamics Review 18(1), 5-45 (2002)

12. Rittgen, P.: Collaborative Modelling Architecture (COMA), http://www.coma.nu/COMA_Tool.pdf (accessed on: 08/05/2009)

13. Hoppenbrouwers, S.J.B.A., van Bommel, P., Jarvinen, A.: Method Engineering as Game Design-An Emerging HCO Perspective on Methods and CASE Tools. In: Halpin, T., et al. (eds.) Workshop Proceedings of EMMSAD 2008 affiliated to CAiSE 2008, Montpellier, France, pp. 97-111 (2008)

14. Goldkuhl, G.: Conversational Analysis as a Theoretical Foundation for Language Action Approaches? In: Weigand, H., Goldkuhl, G., de Moor, A. (eds.) Proceedings of the 8th International Working Conference on LAP (LAP 2003), Tilburg, The Netherlands, July 1-2, pp. 51-69 (2003)

15. Ssebuggwawo, D.: Evaluating Collaborative Modeling Processes: Towards Understanding and Supporting Collaborative Modeling Games. In: Weigand, H., Brinkkemper, S. (eds.) Proceedings of the CAiSE Doctoral Consortium at the CAiSE 2009 Conference, Amsterdam, The Netherlands, June 8-12 (2009) 